\title{
EFFECT OF MATERIAL'S RANDOMNESS ON SCALING OF CRACK PROPAGATION IN CERAMICS
}

\author{
Vadim V. Silberschmidt \\ Wolfson School of Mechanical and Manufacturing Engineering, Loughborough University, LE11 3TU, UK
}

\section{Abstract}

Crack propagation in real (quasi)brittle materials demonstrates various signs of stochasticity; a tortuous character of fracture surfaces, multiple cracking and crack branching observed in experiments are a vivid confirmation of it. Traditional approaches of fracture mechanics represent cracks as geometrically smooth objects with straight (or curved) crack fronts, thus usually neglecting morphology of real cracks. An introduction of a direct account for stochastic features of brittle materials can lead to a more adequate description of real fracture processes. Combining these random features with continuum damage mechanics $(\mathrm{CDM})$ and fracture mechanics, it is possible to study the effect of the material's randomness on crack propagation. CDM describes a macroscopic manifestation of various failure processes developing at lower length scales. The mode-I fracture analysis, based on discretization of the cross-section of specimens containing a sharp notch into rectangular elements (cells), provides detailed information on crack propagation. A necessity to describe a crack with its length changing along its front presupposes a transition from a unique stress-intensity factor to a set of its local values. A computational procedure for simulation of crack-damage interaction and crack propagation in alumina specimens at tension is suggested on the basis of a modification of a lattice scheme unified with ideas of CDM and local stress-intensity factors. Inhomogeneity of material properties is modelled in terms of various random spatial distributions of the initial damage in the specimen's cross-section. A description of complicated morphology of cracks is implemented by means of scaling analysis of the crack-front shape. It is shown that the scaling parameters can be linked to the type of material's randomness.

\section{INTRODUCTION}

Real (quasi)brittle materials usually exhibit - to a different extent - a spatial randomness in their properties. The most common reason for such randomness is non-uniformity in spatial distributions either of material's constituents or of various defects. A macroscopic manifestation of the effect of such randomness on the fracture process is the vivid difference in a crack trajectory and/or in a fragmentation type (number and shape of fragments) of identical specimens of the same material under similar loading conditions. Another sign of it is a well-known scatter in mechanical parameters at the moment of fracture observed for twin specimens of brittle materials. Microscopic variations in distributions of phases or defects result in different scenarios of generation and evolution of failure and should be adequately reflected in modelling schemes for fracture of random media.

In ceramic materials, one of the main microstructural features is the presence of initial porosity (see [1] and references there) caused mainly by manufacturing (e.g. sintering). The random spatial distribution of porosity is responsible for fluctuations in the local stiffness magnitude of ceramics, causing non-uniform stress distributions even under uniform loading conditions. At the same time, pores are nuclei for generation of cracks, the evolution of which is the main mechanism of failure development in ceramics [2]. One of the standard approaches to describe the effect of porosity is to introduce effective elastic coefficients as functions of its level, obtained either experimentally or theoretically [1-4].

The present paper exploits another approach based on a direct introduction of material's randomness using various statistical realisations of the spatial distribution of properties for a single 
specimen. It is the basis for quantitative estimates of various parameters (including scaling ones) for a given combination of a specimen's shape, material properties and loading conditions.

\section{MODEL}

A model for the description of failure evolution in alumina ceramics accounting for their microstructural inhomogeneity was introduced in [5, 6] and applied to the crack propagation process in [7]. It is based on a well-posed CDM approach to failure in brittle materials that has been suggested and discussed in detail in [8]. The proposed approach describes the macroscopic effect of microscopic processes in brittle materials in terms of an additional variable - damage parameter $D$. This parameter characterises the material's deterioration under the applied load due to damage accumulation. The initial damage $D_{0}$ is linked to the manufacturing-induced porosity level, randomly distributed in the material. The spatial randomness of material's properties is directly introduced into the suggested model in terms of the scatter in $D_{0}$ mapped onto a set of cells (elements), into which the area under study is discretized.

The damage evolution law for brittle-elastic materials loaded at tension can be obtained by thermodynamic analysis [8]. Its form for a $2 d$ net of elements that are characterized by a pair of indices $(i, j)$ can be presented in the following form:

$$
D^{i j}=D_{0}^{i j} \exp \frac{E\left(\varepsilon^{i j}\right)^{2}}{2 W^{*}},
$$

where $D^{i j}$ and $D_{0}^{i j}$ are current and initial levels of damage in the element $(i, j), \varepsilon^{i j}$ is a local strain, $E$ is a Young's modulus of the bulk material and $W^{*}$ denotes its continuum damage energy absorption capacity. The latter is a temperature-dependent material property linked to the process energy of damage evolution. The magnitude of this parameter was obtained from the treatment of the experimental data on dynamic failure of alumina at various temperatures in the interval from $20^{\circ} \mathrm{C}$ to $1500^{\circ} \mathrm{C}$, for details see [9].

Three various types of randomness in the initial damage level are considered here: with a uniform probability density function and two normal distributions characterised by various magnitudes of the standard deviation $\gamma$ (Fig. 1). It is worth mentioning that for all types both the distributions' intervals $\left[D_{0 \min }, D_{0 \max }\right]$ for $D_{0}^{i j}$ as well as its mean are the same. The mapping of this interval onto the unit interval $[0,1]$ for parameter $s$ of the probability density functions is performed using the following relation: $s \leftrightarrow\left(D_{0}^{i j}-D_{0 \min }\right) /\left(D_{0 \max }-D_{0 \min }\right)$.

Such introduction of randomness into the modelling scheme results in a non-uniform distribution of stresses over elements even under uniform external loading and in a scatter in the instantaneous levels of damage $D^{i j}$ for the entire set of elements.

Let us consider a traditional case of the initially notched specimen subjected to the mode-I fracture conditions. Consider a rod with a sharp notch, which rectangular cross-section ABCD with dimensions $a \times b$ containing the notch tip EF is discretized into a two-dimensional orthogonal network of $M=N_{x} \times N_{y}$ rectangular elements (Fig. 2). Dimensions of these elements meet the requirements for a representative element being, on the one hand, considerably larger than the characteristic length scale of the microstructure (in our case, the element size is $100 \mu \mathrm{m}$ against the grain size of several micrometers) and, on the other hand, they are small enough to describe macroscopic variations in stress/strain fields. A pair of indices $(i, j)$ denotes here the element situated in the $i$ th column and the $j$ th row of elements, with columns being perpendicular 


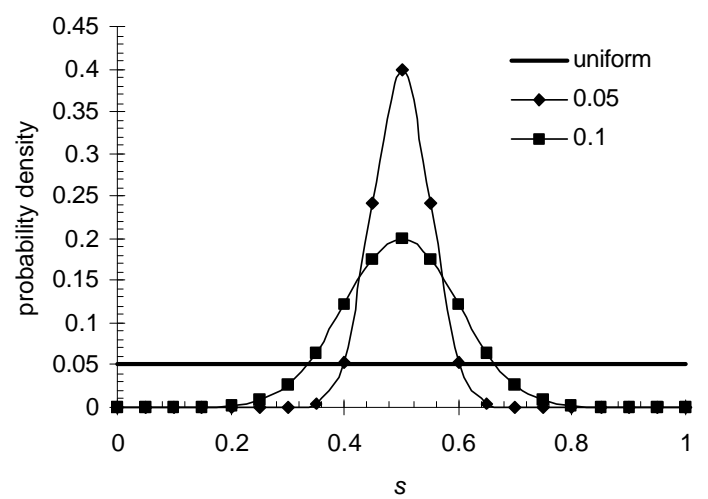

Figure 1: Probability density functions for three studied cases of randomness (numbers stands for respective standard deviations)

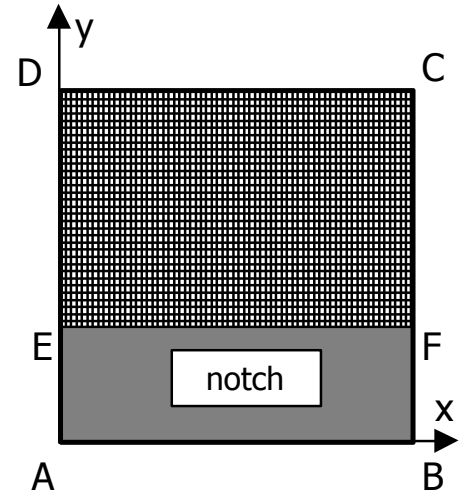

Figure 2: Discretization of the specimen's cross-section, containing the notch's tip

to the notch tip and rows parallel to the $x$-axis (Fig. 2).

The character of the stress distribution over elements under conditions of remote strain for the mode-I crack can be described in the following form:

$$
\sigma^{i j}=K^{i j} E\left(1-D^{i j}\right) \varepsilon^{i j} \text {. }
$$

Equation (2) accounts for the main factors of the case modelled: stress concentration due to the presence of the crack is introduced in terms of stress coefficients $K^{i j}$ and the effect of damage is treated in a traditional CDM way, i.e. material's deterioration is described by the multiplier $\left(1-D^{i j}\right)$. Stress coefficients $K^{i j}$ can be obtained by integrating the well-known relation of linear fracture mechanics $\sigma_{z z}=K_{\mathrm{I}} / \sqrt{2 \pi y}$. Integration should be implemented independently for all columns of elements (the reason for this is given below). As a result, stress coefficients can be presented as

$$
K^{i j}=K_{\mathrm{I}}^{i} \sqrt{\pi / l_{y}}\left(\sqrt{j+1-n_{i}}-\sqrt{j-n_{i}}\right),
$$

where $K_{\mathrm{I}}^{i}$ is a local stress intensity factor (SIF) of the $i$ th column of elements, $l_{y}=b / N_{y}, n_{i}$ is a number of elements occupied by the crack in the $i$ th column. Stress intensity factors for the given geometry and loading conditions can be approximated by respective relations from handbooks of SIF (e.g. [10]):

$$
K_{\mathrm{I}}^{i}=\sqrt{\pi l_{y} n_{i}}\left[0.265\left(1-\bar{n}_{i}\right)^{4}+\frac{0.857+0.265 \bar{n}_{i}}{\left(1-\bar{n}_{i}\right)^{3 / 2}}\right],
$$

where $\bar{n}_{i}=n_{i} / N_{y}$.

Equations (2)-(4) describe stress redistribution processes in elements due to damage and to the presence of a notch. With an increase in external deformation, the growth pace for stresses in 
different elements of the cross-section could vary considerably, especially in the vicinity of the notch tip, resulting in large differences in local stress magnitudes. When the stress level $\sigma^{i j}$ of element $(i, j)$ attains its critical value $\sigma_{\mathrm{m}}^{i j}$ (local strength) linked to the respective damage threshold $D_{\mathrm{m}}^{i j}$, this element fails. This is a local failure criterion for an element: after reaching the state characterized by $\sigma_{\mathrm{m}}^{i j}$ and $D_{\mathrm{m}}^{i j}$ the element's load-bearing capacity vanishes and the accumulated elastic energy $\bar{W}_{\sigma}^{i j}=\left(\sigma_{\mathrm{m}}^{i j}\right)^{2} /(2 E)$ is released. The released energy of this locally failed element is equally distributed to its nearest neighbours causing their transitions to a new state. Details of the algorithm to calculate parameters of this state are given in [6, 7]. A transition of an element to its new state results in increased damage level. If this level fulfils the local failure criterion $D^{i j}=D_{\mathrm{m}}^{i j}$ for the element, a secondary failure process is initiated; even cascades of local failures of elements can occur.

The process of failure of elements adjoining the tip of a crack/notch is a mechanism of crack propagation. Since local failures of elements occur non-simultaneously, various portions along the crack front 'grow' with different rates. It results in a scatter of parameter $n_{i}$ in Eq. (3) along the

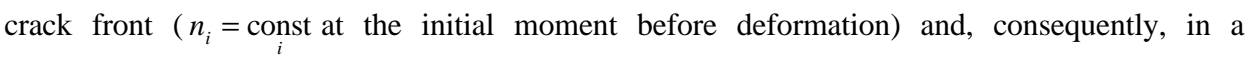
fluctuating level of stress intensity factors, necessitating the introduction of local SIFs.

\section{RESULTS AND DISCUSSION}

The suggested algorithm is used to simulate the damage evolution in specimens of alumina ceramics, loaded by uniform external displacements. The manufacturing-induced porosity is considered to result in the initial average damage of 0.055 , with the upper and lower bounds of its distribution being 0.1 and 0.01 , respectively. The typical calculated load-deformation curve for ? specimen with ? uniform probability density of the initial damage is given in Fig. 3. The straincontrolled loading process ensures stability of calculations for the post-critical behaviour (after

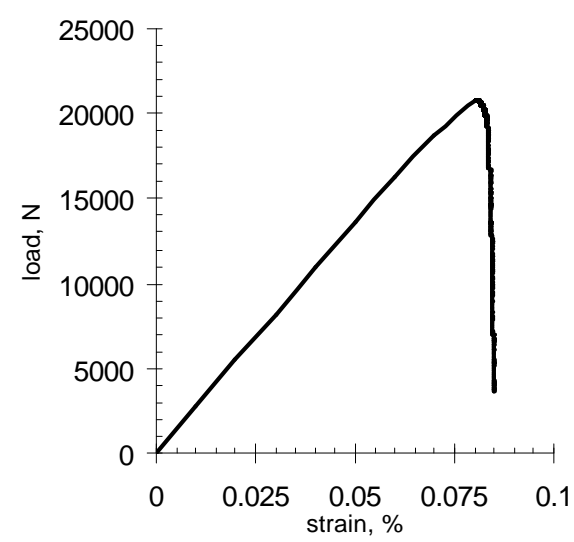

Figure 3: Load-strain curve in case of uniform probability density

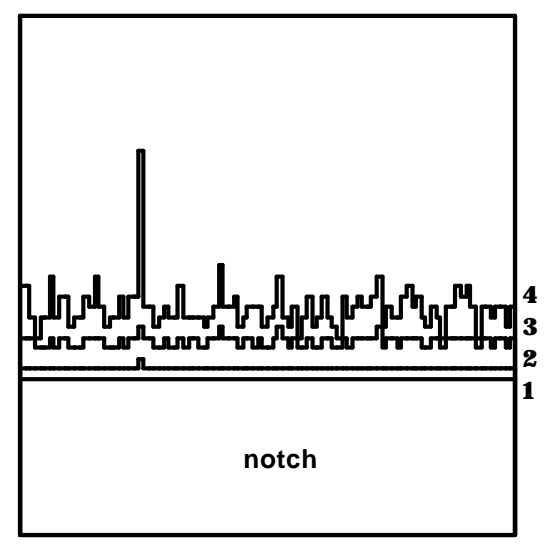

Figure 4: Position of crack front for various deformations: (1) initial position, up to $0.0811 \%$; (2) $0.0825 \%$; (3) $0.083 \%$; (4) $0.0841 \%(\gamma=0.05)$ 
initiation of the crack growth), which is represented by the ascending part of the curve. This part is calculated using several hundreds strain increments, each being $5 \times 10^{-8}$.

The initial randomness in material's properties affect the crack growth process that is linked to non-simultaneous local failure events. The difference in the type of randomness is reflected in specific features of crack propagation in respective specimens. The increase in the distribution's variance results in a larger scatter in the local crack length, with some parts of the crack front considerably outstripping other from the very early stages of the crack growth process. In contrast, these stages in a specimen with $\gamma=0.05$ demonstrate a more ordered (though still random) development: loca1 failures occur in the direct vicinity of the notch tip, and at some moment of deformation the entire row is occupied by the propagated crack, and only after this the first failure occurs in the next row (see curve 2, Fig. 4).

Each statistical realisation of the spatial distribution of initial damage results in the unique scenario of crack propagation and, consequently, morphology of the crack front. A scaling parameter characterising tortuosity of crack fronts can be obtained within the framework of the inplane roughness analysis $[11,12]$, based on the general scheme for characterization of self-affine profiles [13]. The roughness index (also known as the Hurst exponent) $\zeta$ is determined from the scaling relation $y_{\max } \propto r^{\zeta}$, where

$$
y_{\max }(r)=\left.l_{y}\left\langle\left.\max n^{q}\left(r^{\prime}\right)\right|_{x<r^{\prime}<x+r}-\left.\min n^{q}\left(r^{\prime}\right)\right|_{x<r^{\prime}<x+r}\right\rangle\right|_{x} .
$$

Here $y_{\max }(r)$ is a distance along the $y$-axis between the farthest - with respect to the initial notch locally failed element, belonging to a propagating crack, and the closest one within the given window of size $r$ along the crack front. The level of $\zeta$ obtained from microscopic observations was found to be equal $0.60 \pm 0.04$ for $8090 \mathrm{Al}-\mathrm{Li}$ alloy and $0.54 \pm 0.03$ for a Supera2 Ti3Al-based alloy on the length scale from $1 \mu \mathrm{m}$ to $1.5 \mathrm{~mm}$; the detailed analysis of universality of $\zeta$ is given in [11]. The roughness of fracture surfaces (out-of-plane roughness) measured, for instance, by mechanical profilometry is a material-sensitive parameter: for sandstone the roughness exponent $\zeta$ was found to be close to $0.5(0.47 \pm 0.03)$ and for basalt it was higher $(0.80 \pm 0.04)$ for scale length from $25 \mathrm{~mm}$ up to several $\mathrm{cm}$ [14].

Table 1: Roughness exponents and fractal dimension of crack fronts

\begin{tabular}{|c|c|c|c|c|c|}
\hline \multirow{9}{*}{$\begin{array}{c}\text { Stan ard } \\
\text { deviatio / Type } \\
\text { of Rand mness }\end{array}$} & \multirow{3}{*}{$0) 5$} & $\mathrm{~S}$ ain, $\%$ & 0.182 & 0.1835 & 0.1839 \\
\hline & & $\zeta$ & $0.7 \leftleftarrows \longleftarrow 0.06$ & $0.7(\Leftarrow 0.04$ & $0.6] \pm 0.06$ \\
\hline & & $D$ & - & $0.8 \_50.02$ & $0.81 \pm 0.02$ \\
\hline & \multirow{3}{*}{010} & $\mathrm{~S}$ ain, $\%$ & 0.182 & $0 \quad 183$ & 0.1837 \\
\hline & & $\zeta$ & $0.7 \leftleftarrows \longleftarrow 0.03$ & $0.6(\vdash 0.04$ & $0.5 \longleftarrow \pm 0.07$ \\
\hline & & $D$ & - & $0.7\{\llcorner 0.04$ & $0.5 \longleftarrow \pm 0.02$ \\
\hline & \multirow{3}{*}{$\begin{array}{l}\text { Un orm } \\
\text { prob bility } \\
\text { de sity }\end{array}$} & $\mathrm{S}$ ain, $\%$ & 0. 182 & 0.1825 & 0. 183 \\
\hline & & $\zeta$ & $0.5 £ \Leftarrow 0.03$ & $0.5:\llcorner 0.03$ & $0.5 \_ \pm 0.05$ \\
\hline & & $D$ & - & $0.6 \nmid \vdash 0.04$ & $0.5 \longleftarrow \pm 0.05$ \\
\hline
\end{tabular}


Results of numerical simulations based on the introduced description of spatially random brittle alumina with microscopic porosity show the effect of the extent of non-uniformity in material properties on the in-plane roughness (Table 1). There are two obvious trends: (1) crack propagation is characterised by the decreasing roughness exponent; (2) the decrease in the magnitude of the standard deviation causes the increase in $\zeta$. The case of the uniform probability has the lowest levels of the roughness exponent. The same trends are obvious for the fractal dimension of crack fronts, calculated with the use of the box-counting method (see Table 1).

\section{REFERENCES}

[1] R.W. Rice, Porosity of Ceramics. New York e.a.: Marcel Dekker, 1998.

[2] A. Zimmermann and J. Rödel, Generalized Orowan-Petch plot for brittle fracture, J. Am. Ceram. Soc., 81, p. 2527-2532, 1998.

[3] R.G. Munro, Effective medium theory of the porosity dependence of bulk moduli, J. Am. Ceram. Soc., 84, p. 1190-1192, 2001.

[4] M. Kachanov, I. Sevostianov and B. Shafiro, Explicit cross-property correlations for porous materials with anisotropic microstructure, J. Mech. Phys. Solids, 49, p. 1-25, 2001.

[5] J. Najar and V.V. Silberschmidt, Continuum damage and failure evolution in inhomogeneous ceramic rods, Arch. Mech., 50, p. 21-40, 1998.

[6] V.V. Silberschmidt and J. Najar, Computational modelling of the size effect of damage inhomogeneity in ceramics, Comp. Mat. Sci., 13, p. 160-167, 1998.

[7] V.V. Silberschmidt, Crack propagation in random materials: Computational analysis, Comp. Mat. Sci., 26, p. 159-166, 2003.

[8] J. Najar, Continuous damage of brittle solids, in Continuum Damage Mechanics-Theory and Applications, D. Krajcinovic and J. Lemaitre, Editors. New York: Springer, p. 233-294, 1987.

[9] J. Najar, V.V. Silberschmidt and M. Müller-Bechtel, Enhanced fracture energy and effects of temperature at spalling in ceramics: continuum damage modelling, Arch Appl. Mech., 70, p. 145-157, 2000.

[10] H. Tada, P.C. Paris and G.R. Irwin, The Stress Analysis of Cracks Handbook. Del Research Corp., 1973/ASME Press, 2000.

[11] P. Daguier, E. Bouchaud and G. Lapasset, Roughness of a crack front pinned by microstructural obstacles, Europhys. Lett., 31,p. 367-372, 1995.

[12] E. Bouchaud, Scaling properties of cracks, J. Phys.: Condens. Matter, 9, p. 4319-4344, 1997.

[13] J. Feder, Fractals. New York: Plenum Press, 1988.

[14] J.M. Boffa, C. Allain and J.P.Hulin, Experimental analysis of self-affine fractured rock surfaces through shadow length measurements, Physica A, 278, p. 65-86, 2000. 